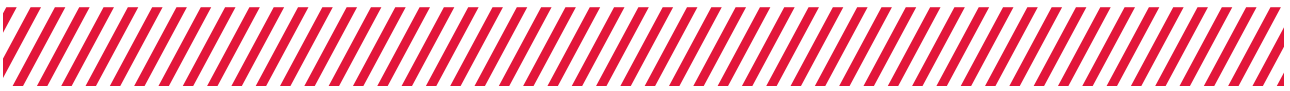

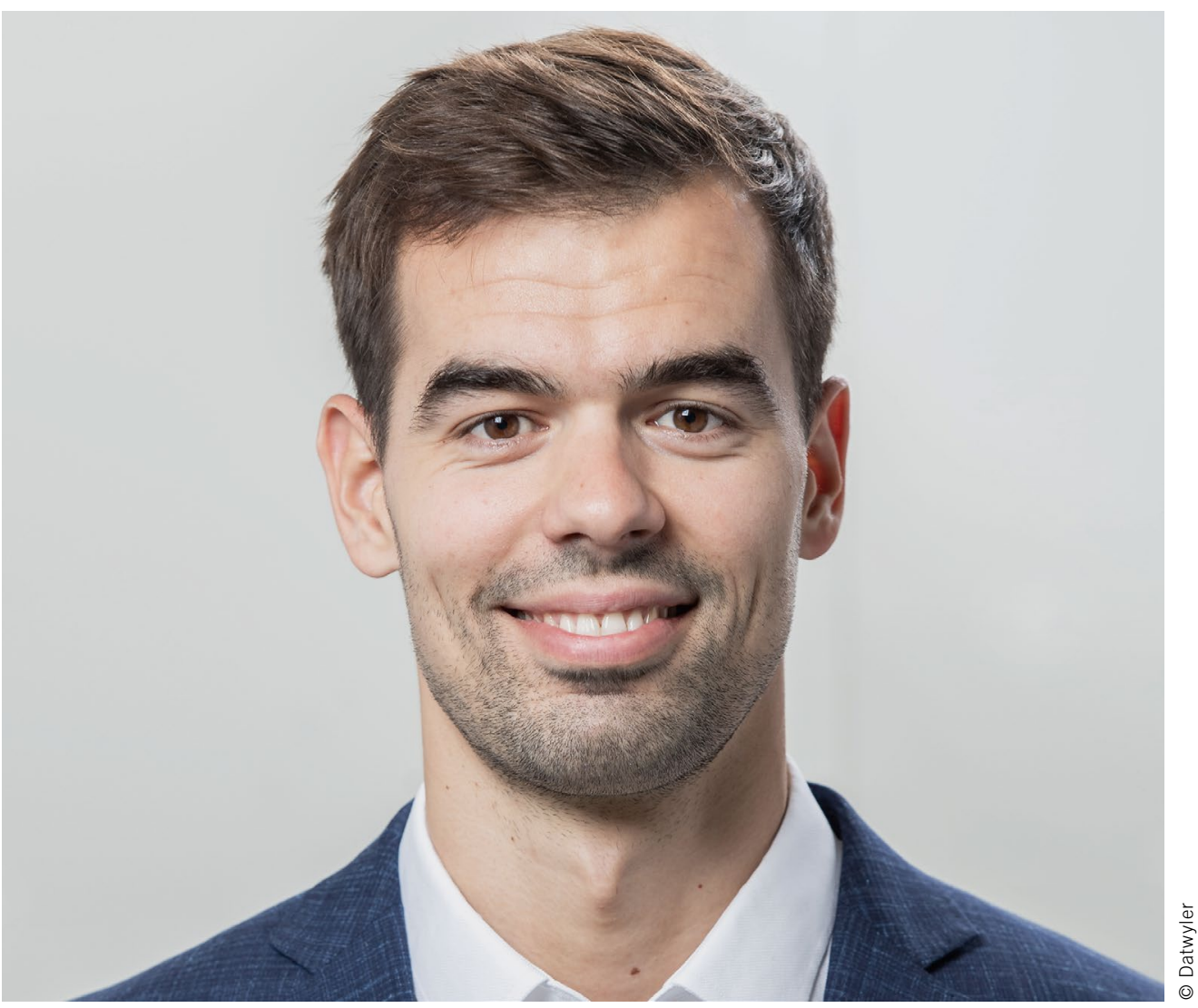

Dr. Jakub Kadlcak

Head of Materials Development and Surface Technologies at Datwyler Mobility

\section{Well Tempering and Sealing of Batteries}

With the rise of electrification, many auxiliary areas will increase in importance, in particular the tempering of batteries. Thermal management in electric vehicles is linked to temperature control of this energy storage in order to keep its optimal working conditions at all times. The temperature of the battery has a direct impact on its performance - sustaining its capacity, affecting the charging time and the state of health in general. At the same time, temperature control is very important for the safety of occupants as overheating and potential thermal runaways can put them at risk.

At Datwyler, we believe thermal management can be improved notably by the intelligent use of the right materials and composites. One of the most effective solutions is to directly immerse the battery in a dielectric fluid. This direct liquid tempering allows the battery to be heated or cooled without the usage of heat exchangers. This contributes to the efficiency of the battery, while reducing its weight and simplifying its design.

For sealing the dielectric fluid, electrically and thermally conductive and electromagnetic shielding materials can be applied, Etemi materials in short. The associated project cov- ers a wide range of sealing material developments - from conventional elastomers to liquid silicon rubber up to thermoplastics. This ensures that customers developing hybrid and battery electric vehicles can realize the full potential of system-critical sealing elements with advanced functionalities. This includes sealing the battery pack with thermally conductive elastomer material to provide structural stability, protecting from the environment and supporting in terms of heat transfer from the battery.

With the traffic sector moving steadfastly toward electric mobility, the focus now is on supporting suppliers and OEMs. At this point, we can offer technical assistance with our material know-how. Through a series of compatibility tests, Datwyler experts can ensure the correct sealing solution can be paired with a number of dielectric fluids - such as with water glycol mixture, perfluoropolyether (PFPE), seed oils - which present a more environmentally friendly alternative - and silicon oils. PFPE may be the main focus for electric mobility in times to come due to its low viscosity and non-flammable qualities. But regardless of which fluid is used, it is vital that sealing solutions are considered early in the development phase. 